\title{
PRODUCTS OF SEVERAL COMMUTATORS IN A LIE NILPOTENT ASSOCIATIVE ALGEBRA
}

\author{
GALINA DERYABINA AND ALEXEI KRASILNIKOV
}

\begin{abstract}
Let $F$ be a field of characteristic $\neq 2,3$ and let $A$ be a unital associative $F$-algebra. Define a left-normed commutator $\left[a_{1}, a_{2}, \ldots, a_{n}\right]\left(a_{i} \in A\right)$ recursively by $\left[a_{1}, a_{2}\right]=a_{1} a_{2}-a_{2} a_{1},\left[a_{1}, \ldots, a_{n-1}, a_{n}\right]=$ $\left[\left[a_{1}, \ldots, a_{n-1}\right], a_{n}\right](n \geq 3)$. For $n \geq 2$, let $T^{(n)}(A)$ be the two-sided ideal in $A$ generated by all commutators $\left[a_{1}, a_{2}, \ldots, a_{n}\right]\left(a_{i} \in A\right)$. Define $T^{(1)}(A)=A$.

Let $k, \ell$ be integers such that $k>0,0 \leq \ell \leq k$. Let $m_{1}, \ldots, m_{k}$ be positive integers such that $\ell$ of them are odd and $k-\ell$ of them are even. Let $N_{k, \ell}=\sum_{i=1}^{k} m_{i}-2 k+\ell+2$. The aim of the present note is to show that, for any positive integers $m_{1}, \ldots, m_{k}$, in general, $T^{\left(m_{1}\right)}(A) \ldots T^{\left(m_{k}\right)}(A) \nsubseteq T^{\left(1+N_{k, \ell}\right)}(A)$. It is known that if $\ell<k$ (that is, if at least one of $m_{i}$ is even) then $T^{\left(m_{1}\right)}(A) \ldots T^{\left(m_{k}\right)}(A) \subseteq T^{\left(N_{k, \ell}\right)}(A)$ for each $A$ so our result cannot be improved if $\ell<k$.

Let $N_{k}=\sum_{i=1}^{k} m_{i}-k+1$. Recently Dangovski has proved that if $m_{1}, \ldots, m_{k}$ are any positive integers then, in general, $T^{\left(m_{1}\right)}(A) \ldots T^{\left(m_{k}\right)}(A) \nsubseteq T^{\left(1+N_{k}\right)}(A)$. Since $N_{k, \ell}=N_{k}-(k-\ell-1)$, Dangovski's result is stronger than ours if $\ell=k$ and is weaker than ours if $\ell \leq k-2$; if $\ell=k-1$ then $N_{k}=N_{k, k-1}$ so both results coincide. It is known that if $\ell=k$ (that is, if all $m_{i}$ are odd) then, for each $A, T^{\left(m_{1}\right)}(A) \ldots T^{\left(m_{k}\right)}(A) \subseteq T^{\left(N_{k}\right)}(A)$ so in this case Dangovski's result cannot be improved.
\end{abstract}

2010 AMS MSC Classification: 16R10, 16R40

Keywords: polynomial identity, product of ideals, commutators

\section{INTRODUCTION}

Let $R$ be an arbitrary unital associative and commutative ring and let $A$ be a unital associative algebra over $R$. Define a left-normed commutator $\left[a_{1}, a_{2}, \ldots, a_{n}\right]\left(a_{i} \in A\right)$ recursively by $\left[a_{1}, a_{2}\right]=a_{1} a_{2}-a_{2} a_{1}$, $\left[a_{1}, \ldots, a_{n-1}, a_{n}\right]=\left[\left[a_{1}, \ldots, a_{n-1}\right], a_{n}\right](n \geq 3)$. For $n \geq 2$, let $T^{(n)}(A)$ be the two-sided ideal in $A$ generated by all commutators $\left[a_{1}, a_{2}, \ldots, a_{n}\right]\left(a_{i} \in A\right)$. Define $T^{(1)}(A)=A$. Clearly, we have

$$
A=T^{(1)}(A) \supseteq T^{(2)}(A) \supseteq T^{(3)}(A) \supseteq \cdots \supseteq T^{(n)}(A) \supseteq \cdots
$$

We are concerned with the following.

Problem 1. Let $k \geq 2$ and let $m_{1}, \ldots, m_{k}$ be positive integers. Find the maximal integer $N=N\left(R, m_{1}, \ldots, m_{k}\right)$ such that, for each $R$-algebra $A$,

$$
T^{\left(m_{1}\right)}(A) \ldots T^{\left(m_{k}\right)}(A) \subseteq T^{(N)}(A) .
$$

Let $X=\left\{x_{1}, x_{2}, \ldots\right\}$ be an infinite countable set and let $R\langle X\rangle$ be the free unital associative algebra over $R$ freely generated by $X$. Define $T^{(n)}=T^{(n)}(R\langle X\rangle)$.

Problem 2. Let $k \geq 2$ and let $m_{1}, \ldots, m_{k}$ be positive integers. Find the maximal integer $N=N\left(R, m_{1}, \ldots, m_{k}\right)$ such that

$$
T^{\left(m_{1}\right)} \ldots T^{\left(m_{k}\right)} \subseteq T^{(N)} .
$$

It is easy to check that Problem 1 is equivalent to Problem 2, and the integer $N$ in both problems is the same.

Problem 2 and some other similar questions have been recently studied by Dangovski [6] (using different terminology). The work of Dangovski was motivated by the results of Etingof, Kim and Ma 9] and Bapat and Jordan [2, which in turn were motivated by the pioneering article by Feigin and Shoikhet [10].

The following assertion was proved by Latyshev [17, Lemma 1] in 1965 (Latyshev's paper was published in Russian) and independently rediscovered by Gupta and Levin [15, Theorem 3.2] in 1983.

Theorem 1.1 (see [15, 17). Let $R$ be an arbitrary unital associative and commutative ring and let $A$ be an associative $R$-algebra. Let $m, n \in \mathbb{Z}, m, n \geq 1$. Then

$$
T^{(m)}(A) T^{(n)}(A) \subseteq T^{(m+n-2)}(A) .
$$


Latyshev [17] has actually proved that $T^{(m)} T^{(n)} \subseteq T^{(m+n-2)}$ in $R\langle X\rangle$; this assertion is equivalent to Theorem 1.1

Note that, for a unital associative ring $R$, we have $\frac{1}{6} \in R$ if and only if $2(=1+1)$ and 3 are invertible in $R$. The theorem below was proved by Sharma and Srivastava [19, Theorem 2.8] in 1990 and independently rediscovered (with different proofs) by Bapat and Jordan [2, Corollary 1.4] in 2013 and by Grishin and Pchelintsev [12, Theorem 1] in 2015.

Theorem 1.2 (see [2, 12, 19]). Let $R$ be an arbitrary unital associative and commutative ring such that $\frac{1}{6} \in R$ and let $A$ be an associative $R$-algebra. Let $m, n \in \mathbb{Z}, m, n>1$ and at least one of the numbers $m, n$ is odd. Then

$$
T^{(m)}(A) T^{(n)}(A) \subseteq T^{(m+n-1)}(A) .
$$

Note that Grishin and Pchelintsev 12 have actually proved that $T^{(m)} T^{(n)} \subseteq T^{(m+n-1)}$; this result is equivalent to Theorem 1.2 .

Let $N_{k}=\sum_{i=1}^{k} m_{i}-k+1$. The proposition below follows immediately from Theorem 1.2,

Proposition 1.3. Let $R$ be an arbitrary unital associative and commutative ring such that $\frac{1}{6} \in R$ and let $A$ be an associative $R$-algebra. Let $k>0$ be an integer and let $m_{i}>0(i=1, \ldots, k)$ be odd integers. Then

$$
T^{\left(m_{1}\right)}(A) \ldots T^{\left(m_{k}\right)}(A) \subseteq T^{\left(N_{k}\right)}(A) .
$$

Let $N_{k, \ell}=\sum_{i=1}^{k} m_{i}-2 k+\ell+2=N_{k}-(k-\ell-1)$. One can deduce from Theorems 1.1 and 1.2 the following proposition (see Dangovski [6, Section 6]).

Proposition 1.4 (see [6]). Let $R$ be an arbitrary unital associative and commutative ring such that $\frac{1}{6} \in R$ and let $A$ be an associative $R$-algebra. Let $k, \ell$ be integers such that $0 \leq \ell<k$. Let $m_{i} \geq 2(i=1, \ldots, k)$ be integers such that $\ell$ of them are odd and $(k-\ell)>0$ of them are even. Then

$$
T^{\left(m_{1}\right)}(A) \ldots T^{\left(m_{k}\right)}(A) \subseteq T^{\left(N_{k, \ell}\right)}(A) .
$$

We prove Proposition 1.4 in Section 2 in order to have the paper more self-contained.

Recently Dangovski [6, Proposition 2.2] has proved a result that can be reformulated as follows.

Theorem 1.5 (see [6]). Let $F$ be a field and let $k$ be a positive integer. Let $m_{1}, \ldots, m_{k}$ be positive integers and let $N_{k}$ be as above. Then there exists an associative F-algebra $A$ such that

$$
T^{\left(m_{1}\right)}(A) \ldots T^{\left(m_{k}\right)}(A) \nsubseteq T^{\left(1+N_{k}\right)}(A) .
$$

One can deduce from Theorem 1.5 the following.

Corollary 1.6. Let $R$ be an arbitrary unital associative and commutative ring and let $k, m_{1}, \ldots, m_{k}, N_{k}$ be as in Theorem 1.5. Then there exists an associative R-algebra A such that (2) holds.

Proof. Suppose that $R$ is not a field. Let $M$ be a maximal ideal of $R$ (by Zorn's lemma, such an ideal $M$ exists). Then $F=R / M$ is a field and the $F$-algebra $A$ of Theorem 1.5 can be viewed in a natural way as an $R$-algebra (with $r \cdot a$ defined by $r \cdot a=(r+M) \cdot a$ for $r \in R, a \in A$ ). Since $A$ satisfies (2), the result follows.

Let $N$ be the integer defined in Problems 1 and 2. If $\frac{1}{6} \in R$ and all the integers $m_{1}, \ldots, m_{k}$ are odd then $N=N_{k}$. Indeed, it follows from Proposition 1.3 and Corollary 1.6 that in this case we always have

$$
T^{\left(m_{1}\right)}(A) \ldots T^{\left(m_{k}\right)}(A) \subseteq T^{\left(N_{k}\right)}(A)
$$

and, in general,

$$
T^{\left(m_{1}\right)}(A) \ldots T^{\left(m_{k}\right)}(A) \nsubseteq T^{\left(1+N_{k}\right)}(A) .
$$

Suppose that $\ell$ of the integers $m_{1}, \ldots, m_{k}$ are odd $(\ell<k)$ and $(k-\ell)>0$ of them are even. Let $\frac{1}{6} \in R$. Then, by Proposition 1.4. $N_{k, \ell} \leq N$ and, by Corollary 1.6. $N \leq N_{k}$. If $\ell=k-1$ (that is, $k-1$ of the integers $m_{1}, \ldots, m_{k}$ are odd and one of them is even) then $N_{k, k-1}=N_{k}$ so $N=N_{k}$. However, if $0 \leq \ell<k-1$ then $N_{k, \ell}=N_{k}-(k-\ell-1)<N_{k}$ so one can only deduce from the results above that $N_{k, \ell} \leq N \leq N_{k}$.

Our main result is as follows.

Theorem 1.7. Let $F$ be a field. Let $k, \ell$ be integers, $0 \leq \ell \leq k$. Let $m_{1}, \ldots, m_{k}$ be positive integers such that $\ell$ of them are odd and $k-\ell$ of them are even and let $N_{k, \ell}$ be as above. Then there exists a unital associative F-algebra A such that

$$
T^{\left(m_{1}\right)}(A) \ldots T^{\left(m_{k}\right)}(A) \nsubseteq T^{\left(1+N_{k, \ell}\right)}(A) .
$$


In a particular case when $k=2$ and $m_{1}, m_{2}$ are even Theorem 1.7 has been recently proved by Grishin and Pchelintsev [12] and independently by the authors of the present article [8]. In another particular case when $m_{1}=m_{2}=\cdots=m_{k-1}=2$ and $m_{k}$ is even this theorem has been proved by Grishin, Tsybulya and Shokola [13, Theorem 3].

The proof of the following result is similar to that of Corollary 1.6 .

Corollary 1.8. Let $R$ be an arbitrary unital associative and commutative ring and let $k, \ell, m_{1}, \ldots, m_{k}, N_{k, \ell}$ be as in Theorem 1.7. Then there exists an associative R-algebra A such that (3) holds.

It follows that if $\frac{1}{6} \in R$ and at least one of the integers $m_{i}$ is even then $N=N_{k, \ell}$ because, by Proposition 1.4 and Corollary 1.8 , in this case we always have

$$
T^{\left(m_{1}\right)}(A) \ldots T^{\left(m_{k}\right)}(A) \subseteq T^{\left(N_{k, \ell}\right)}(A)
$$

but, in general,

$$
T^{\left(m_{1}\right)}(A) \ldots T^{\left(m_{k}\right)}(A) \nsubseteq T^{\left(1+N_{k, \ell}\right)}(A) .
$$

Thus, the solution of Problems 1 and 2 (for $R$ that contains $\frac{1}{6}$ ) is as follows. Let $R$ be a unital associative and commutative ring such that $\frac{1}{6} \in R$ and let $k, m_{1}, \ldots, m_{k}$ be positive integers. Then

$$
N= \begin{cases}N_{k}=\sum_{i=1}^{k} m_{i}-k+1 & \text { if all integers } m_{i} \text { are odd (Dangovski [6]); } \\
N_{k, \ell}=\sum_{i=1}^{k} m_{i}-2 k+\ell+2 & \begin{array}{l}
\text { if } \ell<k \text { of the integers } m_{i} \text { are odd and } \\
k-\ell \text { of them are even. }
\end{array}\end{cases}
$$

Recall that an associative algebra $A$ is Lie nilpotent of class at most $c$ if $\left[u_{1}, \ldots, u_{c}, u_{c+1}\right]=0$ for all $u_{i} \in A$. Theorem 1.7 follows immediately from the following result.

Theorem 1.9. Under the hypotheses of Theorem 1.7, there exists a unital associative F-algebra A such that the following two conditions are satisfied:

i) $T^{\left(1+N_{k, \ell}\right)}(A)=0$, that is, the algebra $A$ is Lie nilpotent of class at most $N_{k, \ell}$;

ii) there are $v_{i j} \in A$ such that

$$
\left[v_{11}, \ldots, v_{1 m_{1}}\right] \ldots\left[v_{k 1}, \ldots, v_{k m_{k}}\right] \neq 0 .
$$

To prove Theorem 1.9 we use the same algebra $A$ that was used in [8, Theorem 1.4].

Remarks. 1. Both Theorem 1.5 and Theorem 1.7 are valid for arbitrary $k$-tuples $m_{1}, m_{2}, \ldots, m_{k}$ of positive integers. However, if $\ell=k$ (that is, if all $m_{i}$ are odd) then Theorem 1.5 gives a stronger result than Theorem 1.7 because $N_{k, k}=N_{k}+1>N_{k}$ and therefore $T^{\left(1+N_{k, k}\right)}(A) \subset T^{\left(1+N_{k}\right)}(A)$. If $\ell=k-1$ (that is, if one of the integers $m_{1}, m_{2}, \ldots, m_{k}$ is even and $k-1$ of them are odd) then $N_{k, k-1}=N_{k}$ so the results of Theorem 1.5] and Theorem 1.7 coincide; and if $\ell<k-1$ (that is, if two or more of the integers $m_{1}, m_{2}, \ldots, m_{k}$ are even) then $N_{k, \ell}=N_{k}-(k-\ell-1)<N_{k}$ so Theorem 1.5 gives a weaker result than Theorem 1.7

2. The proofs of Theorem 1.2 given in [2, [12] and [19] are valid for algebras over an associative and commutative unital ring $R$ such that $\frac{1}{6} \in R$. However, the proof given in [2] can be slightly modified to become also valid over any $R$ such that $\frac{1}{3} \in R$ (see [1, Remark 3.9] for explanation). Moreover, for some specific $m$ and $n$ Theorem 1.2 holds over an arbitrary ring $R$ : for instance, $T^{(3)}(A) T^{(3)}(A) \subset T^{(5)}(A)$ for any algebra $A$ over any associative and commutative unital ring $R$ (see [5. Lemma 2.1]). However, in general Theorem 1.2 fails over $\mathbb{Z}$ and over a field of characteristic 3: it was shown in [7, 16] that in this case $T^{(3)} T^{(2)} \nsubseteq T^{(4)}$ and moreover, $T^{(3)}\left(T^{(2)}\right)^{\ell} \nsubseteq T^{(4)}$ for all $\ell \geq 1$.

3. In 1978 Volichenko proved Theorem 1.2 for $m=3$ and arbitrary $n$ in the preprint 20] written in Russian. In 1985 Levin and Sehgal [18 independently rediscovered Volichenko's result. More recently Etingof, Kim and Ma 9] and Gordienko [11] have independently proved this theorem for small $m$ and $n$; these authors were unaware of the results of [18, 20].

\section{Proofs of Proposition 1.4 and Theorem 1.9}

Proof of Proposition 1.4. Induction on $k$. If $k=1$ then $\ell=0$ so $N_{1,0}=m_{1}$ and (1) holds.

Suppose that $k>1$ and for all products of less than $k$ terms $T^{\left(m_{i}\right)}(A)$ the proposition has already been proved. We split the proof in 3 cases. 
Case 1. Suppose that $m_{k}$ is odd. Then for some $i$ such that $1 \leq i<k$ the number $m_{i}$ is even so we can apply the induction hypothesis to the product $T^{\left(m_{1}\right)}(A) \ldots T^{\left(m_{k-1}\right)}(A)$. By this hypothesis,

$$
T^{\left(m_{1}\right)}(A) \ldots T^{\left(m_{k-1}\right)}(A) \subseteq T^{\left(N^{\prime}\right)}(A)
$$

where $N^{\prime}=\sum_{i=1}^{k-1} m_{i}-2(k-1)+(\ell-1)+2=\sum_{i=1}^{k-1} m_{i}-2 k+\ell+3$. By Theorem 1.2

$$
T^{\left(N^{\prime}\right)}(A) T^{\left(m_{k}\right)}(A) \subseteq T^{\left(N^{\prime}+m_{k}-1\right)}(A)=T^{\left(N_{k, \ell}\right)}(A)
$$

since $N^{\prime}+m_{k}-1=\sum_{i=1}^{k} m_{i}-2 k+\ell+2=N_{k, \ell}$. Thus, in this case (1) holds, as required.

Case 2. Suppose that $m_{k}$ is even and, for some $i$ such that $1 \leq i<k, m_{i}$ is also even. Then we can apply the induction hypothesis to the product $T^{\left(m_{1}\right)}(A) \ldots T^{\left(m_{k-1}\right)}(A)$ so

$$
T^{\left(m_{1}\right)}(A) \ldots T^{\left(m_{k-1}\right)}(A) \subseteq T^{\left(N^{\prime \prime}\right)}(A)
$$

where $N^{\prime \prime}=\sum_{i=1}^{k-1} m_{i}-2(k-1)+\ell+2=\sum_{i=1}^{k-1} m_{i}-2 k+\ell+4$. By Theorem 1.1 .

$$
T^{\left(N^{\prime \prime}\right)}(A) T^{\left(m_{k}\right)}(A) \subseteq T^{\left(N^{\prime \prime}+m_{k}-2\right)}(A)=T^{\left(N_{k, \ell}\right)}(A)
$$

since $N^{\prime \prime}+m_{k}-2=\sum_{i=1}^{k} m_{i}-2 k+\ell+2=N_{k, \ell}$. Hence, in this case (11) holds, as required.

Case 3. Suppose that $m_{k}$ is even and all $m_{i}$ for $1 \leq i<k$ are odd. Applying Theorem $1.2 k-1$ times, we get

$$
T^{\left(m_{1}\right)}(A) \ldots T^{\left(m_{k-1}\right)}(A) T^{\left(m_{k}\right)}(A) \subseteq T^{\left(m_{1}+\cdots+m_{k}-k+1\right)}(A)=T^{\left(N_{k, k-1}\right)}(A)
$$

since $\sum_{i=1}^{k} m_{i}-k+1=\sum_{i=1}^{k} m_{i}-2 k+(k-1)+2=N_{k, k-1}$. Thus, in this case (1) also holds.

The proof of Proposition 1.4 is completed.

The proof of Theorem [1.9 below is a modification of the proof of [8, Theorem 1.4]. First we need some auxiliary results.

Let $G$ and $H$ be unital associative algebras over a field $F$ such that $\left[g_{1}, g_{2}, g_{3}\right]=0,\left[h_{1}, h_{2}, h_{3}\right]=0$ for all $g_{i} \in G, h_{j} \in H$. Note that each commutator $\left[g_{1}, g_{2}\right]\left(g_{i} \in G\right)$ is central in $G$, that is, $\left[g_{1}, g_{2}\right] g=g\left[g_{1}, g_{2}\right]$ for each $g \in G$. Similarly, each commutator $\left[h_{1}, h_{2}\right]\left(h_{j} \in H\right)$ is central in $H$. The following lemma has been proved in [8. Lemma 2.1] by induction on $n$.

Lemma 2.1 (see [8]). Let

where $\ell \geq 2, g_{i} \in G, h_{j} \in H$. Then

$$
c_{\ell}=\left[g_{1} \otimes h_{1}, g_{2} \otimes h_{2}, \ldots, g_{\ell} \otimes h_{\ell}\right]
$$

$$
\begin{aligned}
c_{2}= & {\left[g_{1}, g_{2}\right] \otimes h_{1} h_{2}+g_{2} g_{1} \otimes\left[h_{1}, h_{2}\right], } \\
c_{2 n}= & {\left[g_{1}, g_{2}\right]\left[g_{3}, g_{4}\right] \ldots\left[g_{2 n-1}, g_{2 n}\right] \otimes\left[h_{1} h_{2}, h_{3}\right]\left[h_{4}, h_{5}\right] \ldots\left[h_{2 n-2}, h_{2 n-1}\right] h_{2 n} } \\
+ & {\left[g_{2} g_{1}, g_{3}\right]\left[g_{4}, g_{5}\right] \ldots\left[g_{2 n-2}, g_{2 n-1}\right] g_{2 n} \otimes\left[h_{1}, h_{2}\right]\left[h_{3}, h_{4}\right] \ldots\left[h_{2 n-1}, h_{2 n}\right] } \\
& (n>1), \\
c_{2 n+1}= & {\left[g_{1}, g_{2}\right]\left[g_{3}, g_{4}\right] \ldots\left[g_{2 n-1}, g_{2 n}\right] g_{2 n+1} \otimes\left[h_{1} h_{2}, h_{3}\right]\left[h_{4}, h_{5}\right] \ldots\left[h_{2 n}, h_{2 n+1}\right] } \\
+ & {\left[g_{2} g_{1}, g_{3}\right]\left[g_{4}, g_{5}\right] \ldots\left[g_{2 n}, g_{2 n+1}\right] \otimes\left[h_{1}, h_{2}\right]\left[h_{3}, h_{4}\right] \ldots\left[h_{2 n-1}, h_{2 n}\right] h_{2 n+1} } \\
& (n \geq 1) .
\end{aligned}
$$

Corollary 2.2 (see [8]). Suppose that

$$
\left[f_{1}, f_{2}\right] \ldots\left[f_{2 n-1}, f_{2 n}\right]=0 \quad \text { for all } f_{j} \in H .
$$

Then for all $u_{i} \in G \otimes H$ we have

$$
\left[u_{1}, u_{2}, \ldots, u_{2 n+1}\right]=0 .
$$

Proof. It follows from (44) and Lemma 2.1 that $\left[g_{1} \otimes h_{1}, g_{2} \otimes h_{2}, \ldots, g_{2 n+1} \otimes h_{2 n+1}\right]=0$ for all $g_{i} \in G, h_{j} \in H$. Since each $u_{i} \in G \otimes H$ is a sum of products of the form $g \otimes h(g \in G, h \in H)$, we have $\left[u_{1}, u_{2}, \ldots, u_{2 n+1}\right]=0$ for all $u_{i} \in G \otimes H$, as required.

The following assertion follows immediately from Lemma 2.1

Corollary 2.3. Let $v_{1}=g_{1} \otimes 1, v_{i}=g_{i} \otimes h_{i}\left(i=2, \ldots, 2 m^{\prime}-1\right), v_{2 m^{\prime}}=g_{2 m^{\prime}} \otimes 1$ and let $w_{1}=g_{1}^{\prime} \otimes 1$, $w_{j}=g_{j}^{\prime} \otimes h_{j}^{\prime}\left(j=2, \ldots, 2 n^{\prime}+1\right)$ where $g_{i}, g_{i}^{\prime} \in G, h_{j}, h_{j}^{\prime} \in H$. Then

$$
\begin{aligned}
{\left[v_{1}, \ldots, v_{2 m^{\prime}}\right] } & =\left[g_{1}, g_{2}\right] \ldots\left[g_{2 m^{\prime}-1}, g_{2 m^{\prime}}\right] \otimes\left[h_{2}, h_{3}\right] \ldots\left[h_{2 m^{\prime}-2}, h_{2 m^{\prime}-1}\right], \\
{\left[w_{1}, \ldots, w_{2 n^{\prime}+1}\right] } & =\left[g_{1}^{\prime}, g_{2}^{\prime}\right] \ldots\left[g_{2 n^{\prime}-1}^{\prime}, g_{2 n^{\prime}}^{\prime}\right] g_{2 n^{\prime}+1} \otimes\left[h_{2}^{\prime}, h_{3}^{\prime}\right] \ldots\left[h_{2 n^{\prime}}^{\prime}, h_{2 n^{\prime}+1}^{\prime}\right] .
\end{aligned}
$$


Proof of Theorem 1.9. Two cases are to be considered: the case when $\operatorname{char} F \neq 2$ and the case when $\operatorname{char} F=2$.

Case 1. Suppose that $F$ is a field of characteristic $\neq 2$. Let $E$ be the unital infinite-dimensional Grassmann (or exterior) algebra over $F$. Then $E$ is generated by the elements $e_{i}(i=1,2, \ldots)$ such that $e_{i} e_{j}=-e_{j} e_{i}$, $e_{i}^{2}=0$ for all $i, j$ and the set

$$
\mathcal{B}=\left\{e_{i_{1}} e_{i_{2}} \ldots e_{i_{k}} \mid k \geq 0, i_{1}<i_{2}<\cdots<i_{k}\right\}
$$

forms a basis of $E$ over $F$. It is well known and easy to check that $\left[g_{1}, g_{2}, g_{3}\right]=0$ for all $g_{i} \in E$.

Recall that the $r$-generated unital Grassmann algebra $E_{r}$ is the unital subalgebra of $E$ generated by $e_{1}, e_{2}, \ldots, e_{r}$. Note that $\left[h_{1}, h_{2}, h_{3}\right]=0$ for all $h_{j} \in E_{r}$.

Take $A=E \otimes E_{r}$ where $r=\sum_{i=1}^{k} m_{i}-2 k+\ell=N_{k, \ell}-2$. It is easy to check that $r$ is an even integer. We can apply Lemma 2.1 and Corollaries 2.2 and 2.3 for $G=E, H=E_{r}$.

Note that $\left[f_{1}, f_{2}\right] \ldots\left[f_{r+1}, f_{r+2}\right]=0$ for all $f_{i} \in E_{r}$. Indeed, for all $f, f^{\prime} \in E_{r}$ the commutator $\left[f, f^{\prime}\right]$ belongs to the linear span of the set $\left\{e_{i_{1}} \ldots e_{i_{2 \ell}} \mid \ell \geq 1,1 \leq i_{s} \leq r\right\}$. Hence, $\left[f_{1}, f_{2}\right] \ldots\left[f_{r+1}, f_{r+2}\right]$ belongs to the linear span of the set $\left\{e_{i_{1}} \ldots e_{i_{2 \ell}} \mid \ell \geq(r+2) / 2,1 \leq i_{s} \leq r\right\}$. Since $2 \ell \geq r+2>r$, each product $e_{i_{1}} \ldots e_{i_{2 \ell}}$ above contains equal terms $e_{i_{s}}=e_{i_{s^{\prime}}}\left(s<s^{\prime}\right)$ and, therefore, is equal to 0 . Thus, $\left[f_{1}, f_{2}\right] \ldots\left[f_{r+1}, f_{r+2}\right]=0$, as claimed.

Since $N_{k, \ell}=r+2$, we have $\left[f_{1}, f_{2}\right] \ldots\left[f_{\left(N_{k, \ell}-1\right)}, f_{N_{k, \ell}}\right]=0$ for all $f_{i} \in E_{r}$. Hence, by Corollary 2.2 we have $\left[u_{1}, \ldots, u_{\left(1+N_{k, \ell}\right)}\right]=0$ for all $u_{i} \in A=E \otimes E_{r}$, that is, $T^{\left(1+N_{k, \ell}\right)}(A)=0$, as required.

Now it suffices to find elements $v_{i j} \in A$ such that

$$
\left[v_{11}, \ldots, v_{1 m_{1}}\right] \ldots\left[v_{k 1}, \ldots, v_{k m_{k}}\right] \neq 0 .
$$

Let

$$
\mathcal{P}=\left\{(i, j) \mid 1 \leq i \leq k ; 1 \leq j \leq m_{i}\right\} .
$$

Note that $v_{i j}$ appears in (5) if and only if $(i, j) \in \mathcal{P}$. Let $\mathcal{N}=\sum_{i=1}^{k} m_{i}$ and let $\mu: \mathcal{P} \rightarrow\{1,2, \ldots, \mathcal{N}\}$ be a bijection. Define

Note that

$$
e_{i j}=e_{\mu(i, j)} \quad((i, j) \in \mathcal{P}) .
$$

$$
\prod_{(i, j) \in \mathcal{P}} e_{i j}=(-1)^{\delta} e_{1} e_{2} \ldots e_{\mathcal{N}}
$$

for some $\delta \in\{0,1\}$.

Let $\mathcal{P}^{\prime} \subset \mathcal{P}$,

$$
\mathcal{P}^{\prime}=\left\{\left(i^{\prime}, j^{\prime}\right) \mid 1 \leq i^{\prime} \leq k ; 2 \leq j^{\prime} \leq m_{i}-1 \text { if } m_{i} \text { is even; } 2 \leq j^{\prime} \leq m_{i} \text { if } m_{i} \text { is odd }\right\} .
$$

Let $\mu^{\prime}: \mathcal{P}^{\prime} \rightarrow\left\{1,2, \ldots, \sum_{i=1}^{k} m_{i}-2 k+\ell\right\}=\{1,2, \ldots, r\}$ be a bijection. Define

$$
e_{i^{\prime} j^{\prime}}^{\prime}=e_{\mu^{\prime}\left(i^{\prime}, j^{\prime}\right)} \quad\left(\left(i^{\prime}, j^{\prime}\right) \in \mathcal{P}^{\prime}\right) .
$$

Note that

$$
\prod_{\left(i^{\prime}, j^{\prime}\right) \in \mathcal{P}^{\prime}} e_{i^{\prime} j^{\prime}}=(-1)^{\delta^{\prime}} e_{1} e_{2} \ldots e_{r}
$$

for some $\delta^{\prime} \in\{0,1\}$.

Define

$$
\begin{aligned}
v_{i 1} & =e_{i 1} \otimes 1 ; \\
v_{i j} & =e_{i j} \otimes e_{i j}^{\prime} \quad\left(1 \leq i \leq k ; 2 \leq j \leq m_{i}-1\right) ; \\
v_{i m_{i}} & = \begin{cases}e_{i m_{i}} \otimes 1 & \text { if } m_{i} \text { is even; } \\
e_{i m_{i}} \otimes e_{i m_{i}}^{\prime} & \text { if } m_{i} \text { is odd. }\end{cases}
\end{aligned}
$$

If $m_{i}$ is even then, by Corollary 2.3 ,

$$
\begin{aligned}
& {\left[v_{i 1}, v_{i 2}, \ldots, v_{i m_{i}}\right] } \\
= & {\left[e_{i 1}, e_{i 2}\right]\left[e_{i 3}, e_{i 4}\right] \ldots\left[e_{i\left(m_{i}-1\right)}, e_{i m_{i}}\right] \otimes\left[e_{i 2}^{\prime}, e_{i 3}^{\prime}\right]\left[e_{i 4}^{\prime}, e_{i 5}^{\prime}\right] \ldots\left[e_{i\left(m_{i}-2\right)}^{\prime}, e_{i\left(m_{i}-1\right)}^{\prime}\right] . }
\end{aligned}
$$

Note that $e_{s t} e_{s^{\prime} t^{\prime}}=-e_{s^{\prime} t^{\prime}} e_{s t}$ for all $s, s^{\prime}, t, t^{\prime}$ so $\left[e_{s t}, e_{s^{\prime} t^{\prime}}\right]=2 e_{s t} e_{s^{\prime} t^{\prime}}$. It follows that if $m_{i}$ is even then

$$
\left[v_{i 1}, v_{i 2}, \ldots, v_{i m_{i}}\right]=2^{m_{i}-1} e_{i 1} e_{i 2} \ldots e_{i m_{i}} \otimes e_{i 2}^{\prime} e_{i 3}^{\prime} \ldots e_{i\left(m_{i}-1\right)}^{\prime} .
$$


If $m_{i}$ is odd then, by Corollary 2.3,

$$
\begin{aligned}
& {\left[v_{i 1}, v_{i 2}, \ldots, v_{i m_{i}}\right] } \\
= & {\left[e_{i 1}, e_{i 2}\right]\left[e_{i 3}, e_{i 4}\right] \ldots\left[e_{i\left(m_{i}-2\right)}, e_{i\left(m_{i}-1\right)}\right] e_{i m_{i}} \otimes\left[e_{i 2}^{\prime}, e_{i 3}^{\prime}\right]\left[e_{i 4}^{\prime}, e_{i 5}^{\prime}\right] \ldots\left[e_{i\left(m_{i}-1\right)}^{\prime}, e_{i m_{i}}^{\prime}\right] } \\
= & 2^{m_{i}-1} e_{i 1} e_{i 2} \ldots e_{i\left(m_{i}-1\right)} e_{i m_{i}} \otimes e_{i 2}^{\prime} e_{i 3}^{\prime} \ldots e_{i\left(m_{i}-1\right)}^{\prime} e_{i m_{i}}^{\prime} .
\end{aligned}
$$

It follows that

$$
\left[v_{11}, \ldots, v_{1 m_{1}}\right] \ldots\left[v_{k 1}, \ldots, v_{k m_{k}}\right]=2^{N_{k}-1} \prod_{i=1}^{k} \prod_{j=1}^{m_{i}} e_{i j} \otimes \prod_{i^{\prime}=1}^{k} \prod_{j^{\prime}=2}^{m_{i^{\prime}}^{\prime}} e_{i^{\prime} j^{\prime}}^{\prime}
$$

where

$$
m_{i^{\prime}}^{\prime}= \begin{cases}m_{i^{\prime}}-1 & \text { if } m_{i^{\prime}} \text { is even; } \\ m_{i^{\prime}} & \text { if } m_{i^{\prime}} \text { is odd }\end{cases}
$$

that is,

$$
\left[v_{11}, \ldots, v_{1 m_{1}}\right] \ldots\left[v_{k 1}, \ldots, v_{k m_{k}}\right]=2^{N_{k}-1} \prod_{(i, j) \in \mathcal{P}} e_{i j} \otimes \prod_{\left(i^{\prime}, j^{\prime}\right) \in \mathcal{P}^{\prime}} e_{i^{\prime} j^{\prime}}^{\prime}
$$

By (66) and (7), we have

$$
\left[v_{11}, \ldots, v_{1 m_{1}}\right] \ldots\left[v_{k 1}, \ldots, v_{k m_{k}}\right]=(-1)^{\delta+\delta^{\prime}} 2^{N_{k}-1} e_{1} e_{2} \ldots e_{\mathcal{N}} \otimes e_{1} e_{2} \ldots e_{r} \neq 0,
$$

as required.

Case 2. Suppose that $F$ is a field of characteristic 2. Let $\mathcal{G}$ be the group given by the presentation

$$
\mathcal{G}=\left\langle y_{1}, y_{2}, \cdots \mid y_{i}^{2}=1,\left(\left(y_{i}, y_{j}\right), y_{k}\right)=1(i, j, k=1,2, \ldots)\right\rangle
$$

where $(a, b)=a^{-1} b^{-1} a b$. Then it is easy to check that $\mathcal{G}$ is a nilpotent group of class 2 so $(a, b) c=c(a, b)$ for all $a, b, c \in \mathcal{G}$ and, therefore, $(a, b c)=(a, c) c^{-1}(a, b) c=(a, b)(a, c)$ (see 8 for more details). It is clear that the quotient group $\mathcal{G} / \mathcal{G}^{\prime}$ is an elementary abelian 2-group so $b^{2} \in \mathcal{G}^{\prime} \subseteq Z(\mathcal{G})$ for all $b \in \mathcal{G}$. It follows that $\left(a, b^{2}\right)=1$ so $(a, b)^{2}=\left(a, b^{2}\right)=1$, that is, $(a, b)=(a, b)^{-1}$. Since $(b, a)=(a, b)^{-1}$, we have $(a, b)=(b, a)$ for all $a, b \in \mathcal{G}$.

Let $(<)$ be an arbitrary linear order on the set $\{(i, j) \mid i, j \in \mathbb{Z}, 0<i<j\}$. The following lemma is well known and easy to check.

Lemma 2.4. Let $a \in \mathcal{G}$. Then a can be written in a unique way in the form

$$
\begin{gathered}
a=y_{i_{1}} \ldots y_{i_{q}}\left(y_{j_{1}}, y_{j_{2}}\right) \ldots\left(y_{j_{2 q^{\prime}-1}}, y_{j_{2 q^{\prime}}}\right) \\
\text { where } q, q^{\prime} \geq 0 ; i_{1}<\cdots<i_{q}, j_{2 s-1}<j_{2 s} \text { for all } s, \\
\left(j_{2 s-1}, j_{2 s}\right)<\left(j_{2 s^{\prime}-1}, j_{2 s^{\prime}}\right) \text { if } s<s^{\prime} .
\end{gathered}
$$

Let $F \mathcal{G}$ be the group algebra of $\mathcal{G}$ over $F$. Let $d_{i j}=\left(y_{i}, y_{j}\right)+1 \in F \mathcal{G}$. Note that $d_{i j}=d_{j i}$ and $d_{i i}=0$ for all $i, j$.

Let $I$ be the two-sided ideal of $F \mathcal{G}$ generated by the set

$$
S=\left\{d_{i_{1} i_{2}} d_{i_{3} i_{4}}+d_{i_{1} i_{3}} d_{i_{2} i_{4}} \mid i_{1}, i_{2}, i_{3}, i_{4}=1,2 \ldots\right\} .
$$

The following two lemmas are well known (see, for instance, [14, Lemma 2.1], [15, Example 3.8]); their proofs can also be found in [8].

Lemma 2.5. For all $u_{1}, u_{2}, u_{3} \in F \mathcal{G}$, we have $\left[u_{1}, u_{2}, u_{3}\right] \in I$.

Lemma 2.6. For all $\ell>0$, we have

$$
\left(\left(y_{1}, y_{2}\right)+1\right)\left(\left(y_{3}, y_{4}\right)+1\right) \ldots\left(\left(y_{2 \ell-1}, y_{2 \ell}\right)+1\right) \notin I .
$$

Since the ideal $I$ is invariant under all permutations of the set $\left\{y_{1}, y_{2}, \ldots\right\}$ of generators of the group $\mathcal{G}$, we have the following.

Corollary 2.7. Let $\ell>0$. Then $\left(\left(y_{i_{1}}, y_{i_{2}}\right)+1\right) \ldots\left(\left(y_{i_{2 \ell-1}}, y_{i_{2 \ell}}\right)+1\right) \notin I$ if all integers $i_{1}, i_{2}, \ldots, i_{2 \ell}$ are distinct.

Now we are in a position to complete the proof of Theorem 1.9. Recall that $r=\sum_{i=1}^{k} m_{i}-2 k+\ell=N_{k, \ell}-2$ is an even integer. Let $\mathcal{G}_{r}$ be the subgroup of $\mathcal{G}$ generated by $y_{1}, \ldots, y_{r}$; let $I_{r}=I \cap F \mathcal{G}_{r}$. Take $G=F \mathcal{G} / I$, $H=F \mathcal{G}_{r} / I_{r}$. Take $A=G \otimes H$. By Lemma 2.5 we can apply Lemma 2.1 and Corollaries 2.2 and 2.3 to $A$. 
We claim that $\left[f_{1}, f_{2}\right] \ldots\left[f_{r+1}, f_{r+2}\right] \in I_{r}$ for all $f_{i} \in F \mathcal{G}_{r}$. Indeed, we may assume without loss of generality that $f_{i} \in \mathcal{G}_{r}$ for all $i$. Since

$$
\begin{aligned}
& {\left[f_{2 s-1}, f_{2 s}\right]=f_{2 s-1} f_{2 s}+f_{2 s} f_{2 s-1} } \\
= & f_{2 s-1} f_{2 s}\left(\left(f_{2 s}, f_{2 s-1}\right)+1\right)=f_{2 s-1} f_{2 s}\left(\left(f_{2 s-1}, f_{2 s}\right)+1\right)
\end{aligned}
$$

(recall that $F$ is a field of characteristic 2 ), we have

$$
\left[f_{1}, f_{2}\right] \ldots\left[f_{r+1}, f_{r+2}\right]=f_{1} f_{2} \ldots f_{r+2}\left(\left(f_{1}, f_{2}\right)+1\right) \ldots\left(\left(f_{r+1}, f_{r+2}\right)+1\right) .
$$

It is clear that, for each $s,\left(f_{2 s-1}, f_{2 s}\right)=\prod_{t} c_{i_{s t} j_{s t}}$ for some commutators $c_{i_{s t} j_{s t}}=\left(y_{i_{s t}}, y_{j_{s t}}\right)$. Let $d_{i_{s t} j_{s t}}=$ $c_{i_{s t} j_{s t}}+1$; then $c_{i_{s t} j_{s t}}=d_{i_{s t} j_{s t}}+1$. We have

$$
\begin{aligned}
& \left(f_{2 s-1}, f_{2 s}\right)+1=\prod_{t} c_{i_{s t} j_{s t}}+1=\left(\prod_{t}\left(d_{i_{s t} j_{s t}}+1\right)\right)+1 \\
= & \prod_{t} d_{i_{s t} j_{s t}}+\cdots+\sum_{t<t^{\prime}} d_{i_{s t} j_{s t}} d_{i_{s t^{\prime}} j_{s t^{\prime}}}+\sum_{t} d_{i_{s t} j_{s t} .}
\end{aligned}
$$

It follows that the product $\left(\left(f_{1}, f_{2}\right)+1\right) \ldots\left(\left(f_{r+1}, f_{r+2}\right)+1\right)$ can be written as a sum of products of the form

$$
d_{q_{1} q_{2}} \ldots d_{q_{2 \ell-1} q_{2 \ell}}=\left(\left(y_{q_{1}}, y_{q_{2}}\right)+1\right) \ldots\left(\left(y_{q_{2 \ell-1}}, y_{q_{2 \ell}}\right)+1\right)
$$

where $2 \ell \geq r+2>r$. Hence, in the product (9) we have $q_{t}=q_{t^{\prime}}$ for some $t<t^{\prime}$.

Note that $d_{j_{1} j_{3}} d_{j_{2} j_{3}} \in I$ for all $j_{1}, j_{2}, j_{3}$ because $d_{j_{1} j_{3}} d_{j_{2} j_{3}}=d_{j_{1} j_{3}} d_{j_{2} j_{3}}+d_{j_{1} j_{2}} d_{j_{3} j_{3}} \in S$. Since $d_{i j}=d_{j i}$ for all $i, j$, we have $d_{i_{1} i_{2}} d_{i_{3} i_{4}} \in I$ if any two of the indices $i_{1}, i_{2}, i_{3}, i_{4}$ coincide. It follows that each product (9) belongs to $I_{r}=I \cap F \mathcal{G}_{r}$ and so does the product $\left(\left(f_{1}, f_{2}\right)+1\right) \ldots\left(\left(f_{r+1}, f_{r+2}\right)+1\right)$. Hence, $\left[f_{1}, f_{2}\right] \ldots\left[f_{r+1}, f_{r+2}\right] \in I_{r}$, as claimed. Since $N_{k, \ell}=r+2$, we have $\left[f_{1}, f_{2}\right] \ldots\left[f_{\left(N_{k, \ell}-1\right)}, f_{N_{k, \ell}}\right] \in I_{r}$ for al $f_{i} \in F \mathcal{G}_{r}$.

For any $u \in F \mathcal{G}$, let $\bar{u}=u+I \in G=F \mathcal{G} / I$. Since one can view the algebra $H=F \mathcal{G}_{r} / I_{r}$ as a subalgebra of $G=F \mathcal{G} / I$, we also write $\bar{u}=u+I_{r} \in H=F \mathcal{G}_{r} / I_{r}$ for $u \in F \mathcal{G}_{r}$.

By the observation above, $\left[\bar{f}_{1}, \bar{f}_{2}\right] \ldots\left[\bar{f}_{\left(N_{k, \ell}-1\right)}, \bar{f}_{N_{k, \ell}}\right]=0$ for all $\bar{f}_{i} \in H$. Hence, by Corollary 2.2 we have $\left[u_{1}, \ldots, u_{\left(1+N_{k, \ell}\right)}\right]=0$ for all $u_{i} \in A=G \otimes H$, that is, $T^{\left(1+N_{k, \ell}\right)}(A)=0$, as required.

Let $\mathcal{P}, \mathcal{P}^{\prime}, \mu$ and $\mu^{\prime}$ be as in Case 1 . Recall that $\mathcal{N}=\sum_{i=1}^{k} m_{i}$. Define

$$
y_{i j}=y_{\mu(i, j)} \quad((i, j) \in \mathcal{P}), \quad y_{i^{\prime} j^{\prime}}^{\prime}=y_{\mu^{\prime}\left(i^{\prime}, j^{\prime}\right)} \quad\left(\left(i^{\prime}, j^{\prime}\right) \in \mathcal{P}^{\prime}\right) .
$$

Define

$$
\begin{aligned}
v_{i 1} & =\bar{y}_{i 1} \otimes 1 ; \\
v_{i j} & =\bar{y}_{i j} \otimes \bar{y}_{i j}^{\prime} \quad\left(1 \leq i \leq k ; 2 \leq j \leq m_{i}-1\right) ; \\
v_{i m_{i}} & = \begin{cases}\bar{y}_{i m_{i}} \otimes 1 & \text { if } m_{i} \text { is even; } \\
\bar{y}_{i m_{i}} \otimes \bar{y}_{i m_{i}}^{\prime} & \text { if } m_{i} \text { is odd. }\end{cases}
\end{aligned}
$$

If $m_{i}$ is even then, by Corollary 2.3 ,

$$
\begin{aligned}
& {\left[v_{i 1}, v_{i 2}, \ldots, v_{i m_{i}}\right] } \\
= & {\left[\bar{y}_{i 1}, \bar{y}_{i 2}\right]\left[\bar{y}_{i 3}, \bar{y}_{i 4}\right] \ldots\left[\bar{y}_{i\left(m_{i}-1\right)}, \bar{y}_{i m_{i}}\right] \otimes\left[\bar{y}_{i 2}^{\prime}, \bar{y}_{i 3}^{\prime}\right]\left[\bar{y}_{i 4}^{\prime}, \bar{y}_{i 5}^{\prime}\right] \ldots\left[\bar{y}_{i\left(m_{i}-2\right)}^{\prime}, \bar{y}_{i\left(m_{i}-1\right)}^{\prime}\right] } \\
= & \bar{y}_{i 1} \bar{y}_{i 2} \bar{y}_{i 3} \ldots \bar{y}_{i m_{i}}\left(\left(\bar{y}_{i 1}, \bar{y}_{i 2}\right)+1\right)\left(\left(\bar{y}_{i 3}, \bar{y}_{i 4}\right)+1\right) \ldots\left(\left(\bar{y}_{i\left(m_{i}-1\right)}, \bar{y}_{i m_{i}}\right)+1\right) \\
\otimes & \bar{y}_{i 2}^{\prime} \bar{y}_{i 3}^{\prime} \ldots \bar{y}_{i\left(m_{i}-1\right)}^{\prime}\left(\left(\bar{y}_{i 2}^{\prime}, \bar{y}_{i 3}^{\prime}\right)+1\right)\left(\left(\bar{y}_{i 4}^{\prime}, \bar{y}_{i 5}^{\prime}\right)+1\right) \ldots\left(\left(\bar{y}_{i\left(m_{i}-2\right)}^{\prime}, \bar{y}_{i\left(m_{i}-1\right)}^{\prime}\right)+1\right)
\end{aligned}
$$

If $m_{i}$ is odd then, by the same corollary,

$$
\begin{aligned}
& {\left[v_{i 1}, v_{i 2}, \ldots, v_{i m_{i}}\right] } \\
= & {\left[\bar{y}_{i 1}, \bar{y}_{i 2}\right]\left[\bar{y}_{i 3}, \bar{y}_{i 4}\right] \ldots\left[\bar{y}_{i\left(m_{i}-2\right)}, \bar{y}_{i\left(m_{i}-1\right)}\right] \bar{y}_{i m_{i}} \otimes\left[\bar{y}_{i 2}^{\prime}, \bar{y}_{i 3}^{\prime}\right]\left[\bar{y}_{i 4}^{\prime}, \bar{y}_{i 5}^{\prime}\right] \ldots\left[\bar{y}_{i\left(m_{i}-1\right)}^{\prime}, \bar{y}_{i m_{i}}^{\prime}\right] } \\
= & \bar{y}_{i 1} \bar{y}_{i 2} \bar{y}_{i 3} \ldots \bar{y}_{i\left(m_{i}-1\right)} \bar{y}_{i m_{i}}\left(\left(\bar{y}_{i 1}, \bar{y}_{i 2}\right)+1\right)\left(\left(\bar{y}_{i 3}, \bar{y}_{i 4}\right)+1\right) \ldots\left(\left(\bar{y}_{i\left(m_{i}-2\right)}, \bar{y}_{i\left(m_{i}-1\right)}\right)+1\right) \\
\otimes & \bar{y}_{i 2}^{\prime} \bar{y}_{i 3}^{\prime} \ldots \bar{y}_{i\left(m_{i}-1\right)}^{\prime} \bar{y}_{i m_{i}}^{\prime}\left(\left(\bar{y}_{i 2}^{\prime}, \bar{y}_{i 3}^{\prime}\right)+1\right)\left(\left(\bar{y}_{i 4}^{\prime}, \bar{y}_{i 5}^{\prime}\right)+1\right) \ldots\left(\left(\bar{y}_{i\left(m_{i}-1\right)}^{\prime}, \bar{y}_{i m_{i}}^{\prime}\right)+1\right)
\end{aligned}
$$

It follows that

$$
\left[v_{11}, \ldots, v_{1 m_{1}}\right] \ldots\left[v_{k 1}, \ldots, v_{k m_{k}}\right]=\bar{y} Q \otimes \bar{y}^{\prime} Q^{\prime}
$$


where

$$
\begin{gathered}
\bar{y}=\prod_{i=1}^{k} \prod_{j=1}^{m_{i}} \bar{y}_{i j}, \quad \bar{y}^{\prime}=\prod_{i^{\prime}=1}^{k} \prod_{j^{\prime}=2}^{m_{i^{\prime}}^{\prime}} \bar{y}_{i^{\prime} j^{\prime}}^{\prime}, \\
m_{i^{\prime}}^{\prime}= \begin{cases}m_{i^{\prime}}-1 & \text { if } m_{i^{\prime}} \text { is even; } \\
m_{i^{\prime}} & \text { if } m_{i^{\prime}} \text { is odd, }\end{cases} \\
Q=\prod_{i=1}^{k} \prod_{j=1}^{\left[\frac{m_{i}}{2}\right]}\left(\left(\bar{y}_{i(2 j-1)}, \bar{y}_{i(2 j)}\right)+1\right), \quad Q^{\prime}=\prod_{i^{\prime}=1}^{k} \prod_{j^{\prime}=1}^{\left[\frac{m_{i^{\prime}}^{\prime}-1}{2}\right]}\left(\left(\bar{y}_{i^{\prime}\left(2 j^{\prime}\right)}^{\prime}, \bar{y}_{i^{\prime}\left(2 j^{\prime}+1\right)}^{\prime}\right)+1\right) .
\end{gathered}
$$

Since $\mu$ is injective, all elements $y_{i(2 j-1)}, y_{i(2 j)}\left(i=1,2, \ldots, k ; j=1,2, \ldots,\left[\frac{m_{i}}{2}\right]\right)$ that appear in $Q$ are distinct elements of the set $\left\{y_{1}, y_{2}, \ldots\right\}$. Hence, by Corollary 2.7 we have $Q \neq 0$ in $G=F \mathcal{G} / I$. Similarly, $Q^{\prime} \neq 0$ in $H=F \mathcal{G}_{r} / I_{r}$. Since $\bar{y}$ and $\bar{y}^{\prime}$ are invertible elements of $G$ and $H$, respectively, we have $\bar{y} Q \otimes \bar{y}^{\prime} Q^{\prime} \neq 0$, that is,

$$
\left[v_{11}, \ldots, v_{1 m_{1}}\right] \ldots\left[v_{k 1}, \ldots, v_{k m_{k}}\right] \neq 0,
$$

as required.

This completes the proof of Theorem 1.9

Remark. Recall that in the proof of Theorem 1.9 we use the same algebra $A$ that was used in the proof of [8. Theorem 1.4]. Note that in both proofs one can choose the algebra $A$ different from one used in our proofs. For example, let $F$ be any field and let $r=m+n-4=2\left(m^{\prime}+n^{\prime}-2\right)$. Let $A=F\langle X\rangle / T^{(3)} \otimes F\left\langle X_{r}\right\rangle / T_{r}^{(3)}$ where $X_{r}=\left\{x_{1}, \ldots, x_{r}\right\}$ and $T_{r}^{(3)}=T^{(3)}\left(F\left\langle X_{r}\right\rangle\right)=T^{(3)} \cap F\left\langle X_{r}\right\rangle$. Then $A$ satisfies the conditions i) and ii) of Theorem 1.9, one can check this using a description of a basis of $F\langle X\rangle / T^{(3)}$ over $F$. Such a description can be deduced, for instance, from [3, Proposition 3.2] or found (if char $F \neq 2$ ) in [4, Proposition 9].

Our choice of the algebra $A$ in the proof of Theorem 1.9 was made with a purpose to have the paper self-contained.

\section{ACKNOWLEDGMENTS}

This work was partially supported by CNPq grant 310331/2015-3 and by RFBR grant 15-01-05823. We thank Victor Petrogradsky for pointing out the references [18, 19].

\section{REFERENCES}

[1] N. Abughazalah, P. Etingof, On properties of the lower central series of associative algebras, J. Algebra Appl. 15 (2016) 1650187 (24 pages). arXiv:1508.00943 [math.RA].

[2] A. Bapat, D. Jordan, Lower central series of free algebras in symmetric tensor categories, J. Algebra 373 (2013) $299-311$. arXiv:1001.1375 [math.RA].

[3] S. Bhupatiraju, P. Etingof, D. Jordan, W. Kuszmaul and J. Li, Lower central series of a free associative algebra over the integers and finite fields, J. Algebra 372 (2012) 251-274. arXiv:1203.1893 [math.RA].

[4] A. Brandão Jr., P. Koshlukov, A. Krasilnikov, E.A. Silva, The central polynomials for the Grassmann algebra, Israel J. Math. 179 (2010) 127-144.

[5] E.A. da Costa, A. Krasilnikov, Relations in universal Lie nilpotent associative algebras of class 4, to appear in Comm. Algebra. DOI: 10.1080/00927872.2017.1347661. arXiv:1306.4294 [math.RA].

[6] R.R. Dangovski, On the maximal containments of lower central series ideals, arXiv:1509.08030 [math.RA].

[7] G. Deryabina, A. Krasilnikov, The torsion subgroup of the additive group of a Lie nilpotent associative ring of class $3, J$. Algebra 428 (2015) 230-255. arXiv:1308.4172 [math.RA].

[8] G. Deryabina, A. Krasilnikov, Products of commutators in a Lie nilpotent associative algebra, J. Algebra 469 (2017) 84-95. arXiv:1509.08890 [math.RA].

[9] P. Etingof, J. Kim, X. Ma, On universal Lie nilpotent associative algebras, J. Algebra 321 (2009) 697-703. arXiv:0805.1909 [math.RA].

[10] B. Feigin, B. Shoikhet, On $[A, A] /[A, A, A]$ and on a $W_{n}$-action on the consecutive commutators of free associative algebras, Math. Res. Lett. 14 (2007) 781-795. arXiv:math/0610410

[11] A.S. Gordienko, Codimensions of commutators of length 4, Russian Math. Surveys 62 (2007) 187-188.

[12] A.V. Grishin, S.V. Pchelintsev, On the centers of relatively free algebras with an identity of Lie nilpotency, Sb. Math. 206 (2015) 1610-1627.

[13] A.V. Grishin, L.M. Tsybulya, A.A. Shokola, On T-spaces and relations in relatively free, Lie nilpotent, associative algebras, J. Math. Sci. (N.Y.) 177 (2011) 868-877.

[14] C.K. Gupta, A.N. Krasilnikov, Some non-finitely based varieties of groups and group representations, Internat. J. Algebra Comput. 5 (1995) 343-365.

[15] N. Gupta, F. Levin, On the Lie ideals of a ring, J. Algebra 81 (1983) 225-231.

[16] A. Krasilnikov, The additive group of a Lie nilpotent associative ring, J. Algebra 392 (2013) 10-22. arXiv:1204.2674][math.RA]. 
[17] V.N. Latyshev, On the finiteness of the number of generators of a T-ideal with an element $\left[x_{1}, x_{2}, x_{3}, x_{4}\right]$, Sibirsk. Mat. Zh. 6 (1965) 1432-1434 (in Russian).

[18] F. Levin, S. Sehgal, On Lie nilpotent group rings, J. Pure Appl. Algebra 37 (1985) 33-39.

[19] R.K. Sharma, J.B. Srivastava, Lie ideals in group rings, J. Pure Appl. Algebra 63 (1990) 67-80.

[20] I.B. Volichenko, The $T$-ideal generated by the element $\left[x_{1}, x_{2}, x_{3}, x_{4}\right]$, Preprint no. 22, Inst. Math. Acad. Sci. Beloruss. SSR (1978) (in Russian).

Department of Computational Mathematics and Mathematical Physics (FS-11), Bauman Moscow State Technical University, 2-nd Baumanskaya Street, 5, 105005 Moscow, Russia

E-mail address: galina_deryabina@mail.ru

Departamento de Matemática, Universidade de Brasília, 70910-900 Brasília, DF, Brasil

E-mail address: alexei@unb.br 